\title{
Movement, Music and Creativity in The Elderly with Dementia
}

\author{
Renata Martinec* and Kristina Lera \\ Department of Motor Disturbances, Croatia \\ *Corresponding author: Renata Martinec, Faculty of Education and Rehabilitation Sciences, Department of Motoric Disturbances, Chronic Disease \\ and Art-Therapies, Croatia
}

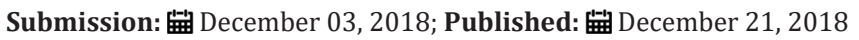

\begin{abstract}
Dementia can have a lot of negative impacts on different functions in the affected person, which can negatively affect the different aspects of quality of life. Statistical data warn us that dementia might become one of the leading medical and social problems in the future. Therefore, significant efforts should be directed to the research and application of various methods of prevention and treatment of this kind of disease. In the context of contemporary concepts, we should keep in mind pharmacological and non-pharmacological approaches. So, in this paper some of the possibilities and the results of using movement, music and creativity are considered. Review of the previous researches shows that their using, based on multimodal sensory stimulation, metaphor, nonverbal expression, imagination and creative experience may have positive influence on social relations, communication, as well on cognitive flexibility. The obtained data contribute to developing of a holistic and integrative idea in the field of treating individuals with dementia.
\end{abstract}

\section{Introduction}

Dementia, as a kind of neurodegenerative disorders, is often connected with the progressive decline of cognitive functions along with a negative influence on motor, emotional and social functioning in persons [1,2]. Dementia decreases quality of life and can often lead to negative psycho-emotional reactions in patients as well as in their family members [1]. It is predicted that in the future dementia maybe one of the leading medical and societal problems. Due to the problems and obstacles that this disease brings to the patient, family members and the wider community, it is necessary to focus on the methods of prevention, therapy and psychosocial support. Even though the usage of pharmacological methods in treating dementia is common, and in some cases extremely necessary, also more emphasis is put on non-pharmacological methods. Non-pharmacological methods can be divided in two separate categories:

standard and alternative/complementary therapies. Both categories of methods have the same goal, which is sensory and cognitive stimulation, as well as reduction of unwanted psychological and behavioral symptoms [3]. Standard methods include cognitive behavioral therapy, reality orientation, reminiscence therapy and validation therapy [4]. Alternative/complementary therapies include different approaches such as activity therapy, pat therapy, acupuncture, aromatherapy, massage, bright-light therapy, sensory gardens, sneeze multi-sensory environments, TENS therapy, expressive art-therapies, etc. [3].

\section{Movement, music and creativity}

Recently, more attention is paid to the usage of movement and psychical activity in the elderly with dementia for the reason that these activities can induce brain plasticity and can alleviate negative cognitive and physical consequences [5]. Different kinds of interventions, such as aerobic, Pilates, body activity for seniors, Thai Chi Chan, flexibility/balance exercises and similar approaches show a positive impact in the improvement of functional capabilities and quality of life. Similarly, they can also contribute to postponement in the decline of executive functions, at least for a shorter period of time [6]. The results of the research show that hippocampus -part of the brain that plays a key role in memory and one of the firsts neurological structures that starts to deteriorate with the progression of dementia - proportionally increases in volume depending on the amount of exercise.

This can lead to the conclusion that the activation of neurohormonal mechanisms through exercise can stimulate and induce cognitive recovery and improvement [7]. However, utilization of these methods has certain limitations. The limitations are connected with the level of preserved motor skills in the patient, as well as their motivation [5]. Thus, in promoting the movement, combinations with other complementary approaches can be proposed to encourage motivation and desire to engage in group activities. As a complementary approach in therapy, expressive art-therapies can also be considered. They are based on the usage of words, music, movement, imagination, creativity, as well as 
expression through visual arts and drama [8]. Since progression of dementia negatively affects the body and mind, it can be assumed that dance movement therapy (DMT) and music therapy (MT) can have a supportive role in their sustaining.

As a type of physical activity, dance can improve physical functioning, while other dance elements can have a positive impact on cognitive and social stimulation. For example, comparison between active dancers and a control group has led to the conclusion that dance exercise and training do not only have a positive impact on posture and balance in dancers, compared to the control group, but as well on the cognition, including memory. It has been confirmed that professional dancers have structurally different sensorimotor networks, as well as structural differences in hippocampus. The results of the research suggest that dance training can contribute to plastic changes on the brain, since brain-imaging studies have shown comprehensive brain activity during the rehearsal of dance movements [5]. Which type of therapy will be used, depends on the needs and capabilities of the patient. Application of the movement therapy with the population who have some sort of physical or intellectual disabilities in the beginning can take the form of interaction that includes small gestures of the hands and palms, with the emphasis on eye contact and facial expression. The sounds that accompany this interaction can only be the sounds produced by the client and/or the therapist, or light music in the background. As the state of the client changes and improves through therapy, the type and complexity of the movement changes as well. The movement and its changes can signify improvement in the clients relationship with him/herself, as well as with the environment [9].

Furthermore, Baird [10] have pointed out, based on the knowledge gained from the previous research, that people with dementia show an interest toward music, as well as that their music memory and ability to react to music are likely preserved even in the later stages of the disease, when verbal communication is already debilitated or lost. The same authors also conclude that music has a unique ability to stimulate and induce the recollection of experiences, as well as positive emotional states. For the dementia patients, that can be a spontaneous and pleasant transition to a state liberated from preoccupations, apathy and confusion.

A number of studies have tried to explain the neuroanatomical changes that can happen as a result of playing and listening to music. For example, some findings suggest that music can affect verbal memory processing in accordance with modification in the left temporal lobe [7-11]. Others state that working memory and attention can be improved by activation of network auditory relay stations in the mid-brain to high-level cortical brain structures and movement-planning areas in the cortex [12]. Not only does music encourage a pleasant mood, but it also has a clear structure and regular changes of musical elements, such as rhythm, melody, tempo, etc., which presumes certain predictability and recognition of the content that follows. This feeling of security and certainty, which is based on auditory perception, can create a foundation for creating new relationships from other sensory modalities. Predictability and appearance of the expected, ensures to the person suffering from dementia an idea that he/she is in a "safe place". In other words, a recognizable routine is certainly an important part of the experience and interpretation of the reality in which the person feels safe and protected.

Due to the decline of cognitive capabilities, especially memory loss, a well-known environment slowly starts to become unrecognizable again, which demands anew learning and adjustment. In that sense, the need for encouragement of creative processes in people with dementia emerges, so they can discover and adopt new strategies for facing the reality. For example, encouraging creative thinking through the usage of expressive art-therapies can stimulate creative solutions in everyday life. According to some authors, these therapies can lead to a number of benefits, such as the feeling of freedom without others' deliberations, development of new skills, social communication, expression of emotion and finding new solutions without the fear of mistake [8-13].

\section{Some specifics of using movement in dementia}

Physical inactivity has largely been seen in people older than 60. The lack of physical activity can contribute to all types of conditions and diseases, such as obesity, cancer, cardiovascular and heart diseases, muscle atrophy, cerebrovascular and respiratory diseases, problems with circulation and feeling of general weakness [14]. Older persons need to be encouraged to participate in physical activities because it can lead to improvements in mood, as well as in respiratory, cardiovascular and locomotor functions [15]. Bearing this in mind, Varghese et al. [16] have researched the impact of different types of leisure activities on the decrease of risk for developing dementia. They conducted their research on a sample of people aged between 75 and 85. Leisure activities that have been included in the research have been separated into two categories: cognitive and physical activities. Cognitive activities included reading, writing, crossword puzzles, board games, organized group discussions or playing musical instruments, while physical activities that were included were golf, tennis, swimming, dancing, exercise, team sports, household chores and babysitting. The results from this research have shown that cognitive activities that have been positively correlated with the lower risk of dementia were reading, playing board games and playing of musical instruments. The only physical activity that had a positive correlation with lower risk of developing dementia was dance. Older people can experience the feeling of vitality through spontaneous expression of movements. That said, some studies have pointed out how dance can have a positive impact on flexibility, strength and muscle endurance, balance and cardiovascular system [14]. Since the population of the elderly differs according to their functional and psychophysical abilities it is crucial that, within the therapeutic framework, those individual abilities and limitations are respected [15].

Different dance themes can be used with the goal to maintain or improve cognitive functions, such as the four seasons, weather, holiday activities, 'here and now' interactions, etc. According to Fersh [15] Movements related to everyday activities, such as movements that represent washing or hanging clothes, can ameliorate motivation for independent functioning. Emotional 
difficulties can also be seen in the patients with dementia. In that case, some parts of the therapeutic program can be directed at processing emotions such as anger, fear, frustration, sorrow, sadness, loneliness, pain, tiredness, guilt and the feelings of worthlessness and abandonment. One of the forms of movement that can be used with people who have dementia is structural improvisation [9]. Structural improvisation is a type of method in which the therapeutic session starts with participants sitting in a circle. The participants begin their therapy with a verbal discussion of their current emotional state and recent positive and negative events in their everyday life. Music is chosen in arrangement with group members. Sometimes the music is barely audible, while in other times, strong rhythm, which helps the participants to synchronize their movements and improves group cooperation and solidarity, is present.

After the initial conversation members of the group sit or stand in the circle while the therapist conducts a short warm-up of the body through movement. The purpose of the warm-up is developing awareness of one's own body and creating a pleasant atmosphere in which group members can feel comfortable enough to move freely through space and explore their bodies through movement [9]. The most crucial element of each session is forwarding the leadership in the group. Each member is asked to perform a movement that the rest of the group will follow and perform. During the movement, the group responds to it with words or with carrying on the movement. The next person in line can produce completely new and nonconnected movements, or they can continue with the previous idea, deliberately or incidentally. The goal of this approach is to encourage motoric activities with a combination of associated mental or emotional images. Those images can induce exploration of new connections and relationships in the imaginative level that can be used in the reality, as well. Circle dancing has been used since the beginning of civilization, through dance accompanied ceremonies and rituals. The circle was used as a means to encourage the sense of community and acceptance [17]. Circle dancing is widely used in DMT and it can include mirroring, reflection of emotional states, retention and physical contact through hand holding. The movements of the participant that are mirrored by the group can have a strong impact on the participant. He or she can see how their emotions and movements echo through the group, which afterwards can lead to the feeling of acceptance and understanding. Spontaneous inducement of kinesthetic empathy between the members of the group can also be seen in this setting [18]. Hand holding can be one of the only active and non-threatening ways in which individuals that are withdrawn or distanced can touch and be touched in a safe setting. For that reason, among others, hand holding, and circle formation are common approaches that are recommended for older adults and especially for the elderly with dementia [19]. Simultaneous usage of multimodal inputs goes hand in hand with sensory stimulation, which, in the DMT setting has the purpose of cognitive stimulation and expression of oneself, while the level of functioning is not important [20]. Animal research has shown how sensory enriched environment has a beneficial effect on brain plasticity and can alleviate cognitive deficits in different neurological disorders [5].
The nature of dance fits the description of an enriched environment for the reason that it combines physical, social and cognitive components, all atonce. It can have an impact on rhythmical and motoric coordination, balance, memory, emotional states, affectivity, social interaction, acoustic and musical stimulation, which leads to the conclusion that dance is an equivalent of sensory stimulation [21]. Use of movement presents a valuable medium in therapy, for the reason that it relies in its great part on non-verbal communication, which can be particularly useful in non-verbal individuals or in those with speech and language difficulties. Individuals with dementia can have a number of accompanying difficulties related to speech production and apprehension, such as aphasia, agnosia and apraxia, which can lead to serious problems in interpersonal communication. Some of the ways in which nonverbal component of communication can be used in DMT is through synchrony and symbolization [22]. Synchrony can be observed through three aspects of body movement: space, rhythm and effort. Symbolization refers to the way in which the emotional experience of the individual is transformed into body movement and bodyexperience. Nystrom [22] conducted a research in which they used DMT as one of the alternative forms of communication.

The purpose of DMT in this scenario was to expand vocabulary repertoire in individuals older than 70 who were living in nursing homes. Through therapy, participants mostly responded through different ways of movement synchronization. In this context, body movements were used to replace speech or in different combinations with speech. For example, when the therapy session was focused on speech dialog, body movements were used to replace words that were necessary. In movement fantasy, additional possibilities of using movement for expression were seen. Another research that was focused on the quality of communication was by Hamill et al. [23]. In their research, the focus was on the connection between using circle in DMT and how can that affect the communication. The results have shown how this type of dance expression had a positive influence on the relationship between individuals affected with dementia and their loved ones, partners and children. Circle dancing gives an opportunity to conduct different types of activities, such as touch, handholding and simultaneous moving through space. For individuals with dementia, who usually have difficulties with time and space orientation, and in some cases their feeling of self is off-balance, this method can assist and support the feeling of integration and connectedness with oneself, as well as one's environment. In that way, it can alleviate fear and isolation, at least for the amount of time the therapy session lasts. According to Karkou [24], DMT can contribute to the postponement of cognitive deterioration, and can ameliorate mood and social interactions of the individual with dementia. It contributes to these positive outcomes through the usage of movement expression, music, relationship between the therapist and the members of the group, and through some specific characteristics that are typical for DMT. Some of those characteristics that can lead to a positive change are embodiment, creativity and improvisation, movement-based improvisation, symbolic and metaphorical movement $[25,26]$. The metaphors used in symbolic movement can also contribute to emotional distancing from unsettling memories and feelings, 
as well as enable safe exploration through movements, which can change cognition and feelings [24].

Research that has been directed to test the differences between DMT and household activities has shown how participants that were included in the DMT program had better abilities at solving visuospatial tasks, planning tasks and had a partial improvement in spoken words [27]. Even though previously mentioned results did not show any positive influence of DMT in the domain of memory, otherwise was seen in the research of Rösler et al. [28]. In their research, they have shown how waltz lessons had a significant positive impact on rhythm, movement flow and implicit memory. These results are encouraging, for the reason that it has been known that implicit memory stimulation can contribute to learning of new skills, or even regeneration of old ones. With the goal of systematically defining the benefits of DMT, Lyons et al. [29] conducted a review of previous research done in this field. Available research articles were analyzed, and it was observed that great importance is given to improvisation, spontaneous expression, rhythmical synchrony and symbolization, as well as to touch, which is thought to be one of the key therapeutic components.

In a great deal of previous studies, mirroring and circle dancing have been mentioned and researched. Most of the studies included in this analysis had a similar design to the dance session, which includes a warm-up, working through a specific relevant topic and a closing ritual. In some of the research different visual, auditive, tactile and kinesthetic stimuli were used. In a larger number of research, the emphasis was on the valuable moments in which individuals with dementia responds in a more integrated way, building connections and relationships between their thoughts, feelings and physical sensations. An interesting concept of using dance movement therapy in practice is the project "Moving memories" launched by the Dance mind Organization [30]. The program that was part of this project was carried out once a week, for over a year. The participants of this program were individuals with different stages of dementia, mostly mild and moderate. In dance sessions there was around 10 to 14 participants as well as 2 to 3 caregivers. The sessions were always held in the same room, to keep the feeling of continuity and security in participants with dementia. During dance therapy sessions, dance and music were used with the goal of achieving embodiment, the development of social interaction and inducing self-expression and communication. The results of this program show how it was effective in managing to prompt the sharing of experiences between participants, the feeling of enjoyment and in facilitating creativity. Authors of this project accentuated the importance of directing the attention on participants, did they participate in the activity and interaction. In other words, it is important to focus on the motivation of the participants and the therapy process itself, and not necessarily on the level of success in execution of activities. Furthermore, even though psychotropic medication is effective in reducing symptoms of dementia, they can also have some negative side effects such as the feeling of drowsiness and inactivity. For that reason, it is important to motivate the participants to take part in the activities, which can be done by making session interesting, fun and interactive.

\section{Specifics of using music in persons with dementia}

Music can give plenty of possibilities as one form of auditive stimulations. Among others, it has the possibility to include emotional and cognitive responses [5]. According to some research, music can have a positive impact on alleviating unwanted behavioral symptoms, stimulating verbal expression and improving social and emotional functioning [24]. The caretakers of individuals with dementia, as well as their family members, stress how listening to music, singing and dance improve and encourage motivation for carrying out different day-to-day activities. As dementia progresses, individuals slowly start to lose the ability to process sensory information and to adjust to the environment's demands. For that reason, some of the consequences of sensory deprivation can be seen, such as repeated movements, primitive vocalization, aggression, wandering and absentness. That said, different interventions in the framework of sensory stimulation could promote adaptive answers to the environment and complex interpersonal interaction. This can be done with the usage of olfactory and tactile props, as well as with music [20]. The therapist often uses sensory stimuli such as different objects, music and sound effects because they can contribute to cognitive stimulation and prompt interaction and connectedness between the group members.

The gestures that clients make while using the objects and props (scarfs, ribbons, etc.) encourage imagination and bring back memories. It is common practice to use music during DMT because their simultaneous combination contributes to greater brain activity. Unlike passive listening to music, the combination of them is surly more cognitively demanding. Comparison between dancing with and without music has shown how dancing with the music in the background activates specific regions of the brain. Dorsal vermis of the cerebellum, putamen and medial geniculate cortex, the regions of the brain that are activated during dancing to music, are thought to be included during synchrony of the movement with the rhythm of the music [31]. Unlike passive music listening, which doesn't demand division of attention to different environmental cues, dancing with music requires processing two different stimuli, in other words, it demands attention to be divided [5]. Based on the results from the previous research in this field, it is possible to define a protocol related to the usage of music within the framework of wholesome psycho-emotional support of individuals with dementia. In that context, certain guidelines can be defined, related to the choice and the way of using musical stimulations. For example, during reminiscence-based music therapy, it is more appropriate to use well-known musical themes and melodies for the reason that they can serve as a trigger towards invocation of past experiences [32]. In addition, while choosing the music type and style, it is important to respect personal preference of the client [33]. Furthermore, the research conducted by Raglio et al. [34], 
has shown how application of MT has greater efficacy in soothing socially unacceptable behavioral symptoms in individuals with dementia, compared to passive listening to the music. Moreover, the results from Holmes et al. [35] show how live music can have immediate and exciting influence in apathetic individuals, no matter the stage of dementia. However, some researches have shown how even passive music listening can lead to a reduction of anxiety and can improve the quality of sleep in persons with dementia [33]. Same authors accentuate the importance of conducting research in the field of guided musical improvisation and its effect on emotional expression, interpersonal communication, and on reducing the feeling of frustration that is the consequence of feeling helpless.

\section{Conclusion}

The term dementia is often bonded with various difficulties of cognitive, emotional and social functioning which negatively influence the quality of life. For that reason, it is crucial to treat those problems with using standard medical procedures, but as well as with non-pharmacological, complementary methods. In recent times, more attention has been given to using arts, movement, expression and musical stimulations, respectively. Review of the previous researches demonstrates the positive influence of multimodal sensory stimulation, metaphor, nonverbal expression, imagination and creative experience on social relations and communication, as well on cognitive flexibility. These approaches could induce regression to the sensorimotor phase, which demand a simpler level of perception and cognition. However, it may serve as foundation for the development of more complex perceptions and reactions. Non-demanding application of movements, dance and music offers the feeling of safety and security to the individual with dementia, which is often disrupted by fear and worry about the progression of disease. Sharing these experiences through group therapy offers the individuals positive experience and the feeling of appreciation and acceptance. It also gives the opportunity to find joy, fulfilment and re-establishing purpose in the individual with dementia. Although various complementary approaches cannot have influence on the final outcome of the disease, they can help to induce a state of comfort and satisfaction, and thus to improve the quality of life in those life moments when the whole world around us becomes a threatening, incomprehensible and unknown place of our existence.

\section{References}

1. Žikić M, Žikić TR, Semnic M, Semnic R, Đajić V, et al. (2009) Epidemiology, etiology, classification, diagnosis of differential diagnosis and-demonstration of dementia. News from neurology, psychiatry and border areas $17(2): 47-52$

2. Osman SE, Tischler V, Schneider J (2016) Singing for the Brain: Aqualitative study exploring the health and well-being benefits of singing for people with dementia and their careers. Dementia 15(6): 1326-1339.

3. Abraha I, Rimland J, Trotta F, Dell' AG, Cruz-Jentoft A (2016) Systematic review of systematic reviews of non-pharmacological interventions to treat behavioral disturbances in older patients with dementia. The SENATOR-On Top series. BMJ Open 7(3): e012759.
4. Douglas S, James I, Ballard C (2004) Non-pharmacological interventions in dementia. Advances in psychiatric treatment 10(3): 171-177.

5. Dhami P, Moreno S, DeSouza JF (2015) New framework for rehabilitation-fusion of cognitive and physical rehabilitation: the hope for dancing. Frontiers in psychology 5: 1478 .

6. McLaren AN, LaMantia MA, Callahan CM (2013) Systematic review of nonpharmacologic intervention to delay functional decline in community dwelling patients with dementia. Aging \& mental health 17(6): 655666

7. Ho R, Cheung J, Chan W, Cheung I, Lam L (2015) A 3-arm randomized controlled trial on the effects of dance movement intervention and exercises on elderly with early dementia. BMC Geriatrics 15(1): 127.

8. Martinec R (2018) Complementary and art-therapies as a supportive approach in gerontology and geriatrics. Gerontology \& geriatrics studies 3(1): 1-4.

9. Stanton K (1991) Dance movement therapy: An introduction. The British journal of occupational therapy 54(3): 108-110.

10. Baird A, Samson S (2015) Music and dementia. Prog Brain Res 217: $207-$ 235.

11. Chan AS, Ho YC, Cheung MC (1998) Music training improves verbal memory. Nature 396(6707): 128

12. Sridharan D, Levitin DJ, Chafe CH, Berger J, Menon V (2007) Neural dynamics of event segmentation in music: converging evidence for dissociable ventral and dorsal networks. Neuron 55(3): 521-532.

13. Tokić R (2017) Creativity in third age. Andragoški glasnik 21(1-2): 7-25.

14. Hwang PW, Braun KL (2015) The Effectiveness of dance interventions to improve older adults' health: A systematic literature review. Alternative therapies in health and medicine 21(5): 64-70.

15. Fersh IE (1982) Dance/movement therapy: A holistic approach to working with the elderly. Activities, adaptation and aging 2(1): 21-30.

16. Verghese J, Lipton RB, Katz MJ, Hall CB, Derby CA, et al. (2003) Leisure activities and the risk of dementia in the elderly. The New England Journal of Medicine 348(25): 2508-2516.

17. Karampoula E, Panhofer H (2018) The circle in dance movement therapy: A literature review. The arts in psychotherapy 58(2018): 27-32.

18. Meekums B (2012) Kinesthetic empathy and movement metaphor in dance movement psychotherapy. In: Reynolds D, Reason M. (Eds.), Kinaesthetic empathy in creative and cultural practices. The University of Chicago Press, pp. 51-67.

19. Koch S, Kunz T, Lykou S, Cruz R (2014) Effects of dance movement therapy and dance on health-related psychological outcomes: A meta-analysis. Arts in Psychotherapy 41(1): 46-64

20. Goldstein Levites N (2006) Dance/movement therapy and sensory stimulation: A holistic approach to dementia care. American journal of dance therapy's 38(2): 429-436.

21. Kattenstroth JC, Kolankowska I, Kalisch T, Dinse HR (2010) Superior sensory, motor, and cognitive performance in elderly individuals with multiyear dancing activities. Front. Aging Neurosci 2: 1-9.

22. Nyström K, Lauritzen SO (2005) Expressive bodies: demented persons communication in a dance therapy contex. Health: An interdisciplinary journal for the social study of health, illness and medicine 9(3): 297-317.

23. Hamill M, Smith L, Röhricht F (2012) 'Dancing down memory lane': Circle dancing as a psychotherapeutic intervention in dementia-a pilot study. Dementia 11(6): 1-16.

24. Karkou V, Meekums B (2017) Dance movement therapy for dementia (Review). Cochrane Database of Systematic Reviews 2: 1-28. 
25. Karkou V, Sanderson P (2006) Arts therapies: A research-based map of the field. Elsevier, Edinburgh, UK

26. Young J, Manthrop C, Howells D, Tullo E (2011) Developing a career communication intervention to support personhood and quality of life in dementia. Ageing and society 23(7): 1003-1025.

27. Hokkanen L, Rantala L, Remes AM, Härkönen B, Viramo P (2008) Dance and movement therapeutic methods in management of dementia: a randomized, controlled study. Journal of the American geriatric society 56(4): 771-712.

28. Rösler A, Seifritz E, Krauchi K, Spoerl D, Brokuslaus I, et al. (2002) Skill learning in patients with moderate Alzheimer's disease: A prospective pilot-study of waltz-lessons. International journal of geriatric psychiatry 17(12): 1155-1156.

29. Lyons S, Karkou V, Roe B, Meekums B, Richards M (2018) What research evidence is there that dance movement therapy improves the health and wellbeing of older adults with dementia? A systematic review and descriptive narrative summary. The arts in psychotherapy 60: 32-40.
30. Coaten R, Heeley T, Spitzer N (2003) Dancemind's 'Moving Memories' evaluation and analysis; a UK based dance and health project for people living with dementia and their care-staff. UNESCO observatory multi-disciplinary journal in the arts 3(3): 1-14.

31. Brown S, Parsons LM (2008) The neuroscience of dance. Scientific American 299(1): 78-83.

32. Ashida S (2000) The effect of reminiscence music therapy sessions on changes in depressive symptoms in elderly persons with dementia. J Music Ther 37(3): 170-182.

33. Balakrishnan RN, Browne W, Marley J, Heim C (2013) Music and dementia. Degenerative Neurological and Neuromuscular Disease 3(3): 47-51.

34. Raglio A, Bellelli G, Traficante D (2008) Efficacy of music therapy in the treatment of behavioral and psychiatric symptoms of dementia. Alzheimer Dis Assoc Disord 22(2):158-162.

35. Holmes C, Knights A, Dean C, Hodkinson S, Hopkins V (2006) Keep music live: music and the alleviation of apathy in dementia subjects. Int Psychogeriatr 18(4): 623-630.

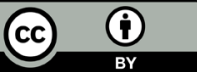

Creative Commons Attribution 4.0 International License

For possible submissions Click Here
Submit Article

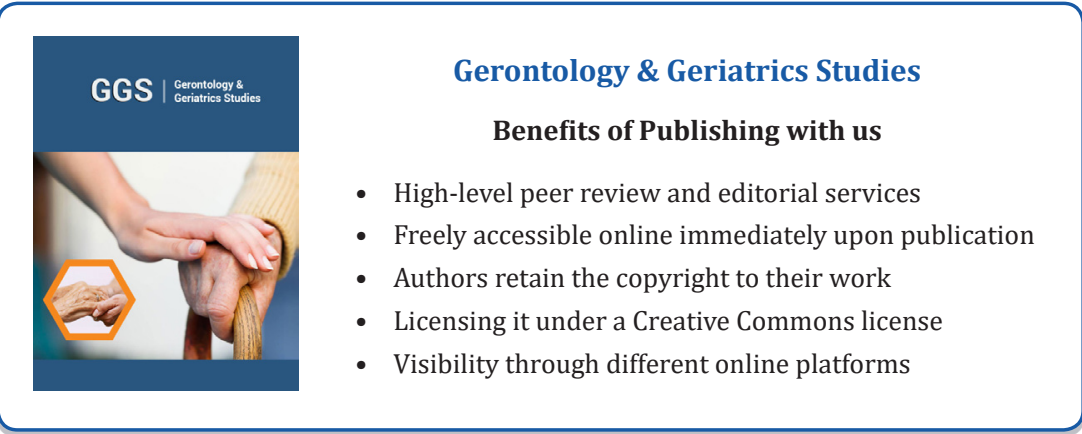

